\title{
Tension in Fluttering Flags
}

\author{
Peter M. Moretti ${ }^{\dagger}$ \\ Oklaboma State University, School of Mechanical \& Aerospace Engineering, Stillwater, OK 74078-5016, USA
}

(Received 22 June 2003; accepted 20 October 2003)

\begin{abstract}
When a flag flutters, tension is dynamically induced by the two-dimensional vibratory motion. The dynamic process, involving centrifugal forces due to the curved path of the trailing edge, is similar to the whipping of an oscillating rope, and accounts for most of the dragforce observed at the flagpole attachment (luff). Conversely, the induced tension, combined with the curvature of the fabric, opposes the pressure forces from the flow field and extracts momentum from it. In order to estimate post-critical flag and panel flutter amplitudes, it is necessary to compute the structural stiffening due to dynamically induced tension. Tension in typical flag flutter motion, consisting of a travelling wave growing in amplitude as it progresses towards the trailing edge (leech), is obtained by approximate analysis, using a computer algebra system. The time-averaged tension depends on the square of the velocity amplitude of the oscillating fabric; the distribution of time-averaged tension is shown for a typical flag flutter motion. An estimate of the tension fluctuations is developed: the fluctuations are small (relative to the average tension) at locations several wavelengths from the leech, but are important near the leech. The general partial differential equation (PDE) of motion is obtained from Hamilton's principle. The inducedtension term in the governing PDE derives from the in-plane kinetic energy of the flag motion. Dynamically induced tension is shown to be important if the stiffness of the fabric is low: an order-of-magnitude criterion is presented.
\end{abstract}

${ }^{\dagger}$ Member of the International Institute of Acoustics and Vibration (IIAV)

\section{INTRODUCTION}

Flag flutter is a challenging problem in physics. The stability limit is very low (flags can be observed to flutter even at very low wind speeds) and yet the flutter amplitudes and modes are steady. The prediction of flag-flutter amplitudes could be a fundamental contribution to fluid/structure interaction. An accurate solution is difficult, and probably impossible without considering the dynamically induced tension terms derived below.

The three fundamental questions raised by flag flutter are: 1. Why does the flag flutter?

2. Why is the drag of a fluttering flag greater than that of a rigid vane?

3. What limits the amplitudes of a fluttering flag?

The first question was answered qualitatively by Thoma, ${ }^{1}$ when he pointed out that flow over a wavy surface produces an elevated pressure in the troughs and a reduced pressure over the crests, tending to increase the amplitudes. Sparenberg confirmed that a travelling wave is generated. ${ }^{2}$

The second question was raised by Fairthorne. ${ }^{3} \mathrm{He}$ observed that the increased drag varies directly with the massper-unit-area of the material, consistent with causation by the dynamics of the motion. Thoma ${ }^{4}$ obtained the average tension in a waving rope and linked it to the third question by pointing out that this dynamically induced tension tends to oppose the fluid-dynamic instability. ${ }^{1}$ Our objective is to study the balance of forces.

When the flag curves, any tension tends to flatten the flag, opposing pressure differences across the flag. These pressure differences are complementary to the flow field around the flag. Conversely, the flow field around the flag generates pressure forces normal to the flag, accelerating the fabric; the resulting motions of the flag generate centrifugal forces which induce tension in the flag; and this tension opposes and ultimately limits the amplitudes of the flag motion.

This process dynamically transform normal (lift) forces on a flexible fabric into tension (drag) forces. It can be studied or measured either from the structural or from the fluid side:

- from the structural side, we can analyse the dynamics of the flag (as we will do below), or measure the drag forces at the flagpole; and

- from the fluid side, we can analyse the flow field, or measure the velocity profile in the wake.

The drag force must, of course, balance the momentum defect in the wake.

For the purpose of the initial physical analysis, the following simplifications will be made:

A. The flag is perfectly flexible: the bending stiffness term is neglected.

B. The flag is inextensible: the path length from luff to leech is constant.

C. Gravity is neglected, and the deflections are uniform across the width.

D. The flag is wide compared to wave-length: the flow-field is two-dimensional.

E. Except for skin-friction calculations, the flow-field is potential flow.

The limitations introduced by these assumptions can be re-examined later.

\section{INITIAL STABILITY}

It has been proposed that flag flutter is caused either by vortex shedding from the flagpole, or else by pressure feedback from the vortex street in the wake of a flat plate or sheet. However, observed flutter does not match either Strouhal frequency. Hence, our analysis looks for an instability phenomenon. 